\title{
Acercando la empresa a la universidad: Una experiencia del uso del método del caso como herramienta pedagógica
}

María Agustina Tauro ${ }^{1}$

Guadalupe Oliveras $^{2}$

\footnotetext{
${ }^{1}$ Facultad de la Micro, Pequeña y Mediana Empresa / Universidad Provincial del Sudoeste

${ }^{2}$ Facultad de la Micro, Pequeña y Mediana empresa / Universidad Provincial del Sudoeste (UPSO)
} 


\section{ACERCANDO LA EMPRESA A LA UNIVERSIDAD: UNA EXPERIENCIA DEL USO DEL MÉTODO DEL CASO COMO HERRAMIENTA PEDAGÓGICA}

\section{Resumen}

El trabajo narra la experiencia de un grupo de docentes de la Universidad Provincial del Sudoeste (UPSO) sobre la enseñanza de la creación y gestión de pequeñas empresas a estudiantes universitarios, utilizando como herramienta pedagógica el método del caso. Para poder redactar los casos se consideraron las particularidades de las ciudades en las cuáles se dictan clases, la impronta de la Universidad que brinda carreras universitarias con perfil emprendedor, así como la orientación de las distintas carreras que se dictan. Del trabajo surge una compilación de 13 casos de MiPyMEs del Sudoeste de la Provincia de Buenos Aires (SOB), de diferentes sectores, junto con una serie de actividades para la aplicación de los docentes de la institución. Se logra desarrollar una herramienta de trabajo adecuada al contexto en el que se trabaja, el SOB, muy diferente al ecosistema empresarial que quizás se puede encontrar en las grandes metrópolis.

Palabras clave: Método del caso. Emprendedorismo. MiPyMEs. Universidad. 


\section{Introducción}

La Universidad Provincial del Sudoeste (UPSO) se encuentra ubicada en el Sudoeste de la Provincia de Buenos Aires (SOB), Argentina, y nace en un período de replanteo y discusión acerca del nuevo rol que debiera desempeñar la Universidad. Desde su gestación, se plantea como objetivo contribuir al desarrollo armónico de su región de influencia, tomando y completando un programa educativo con características propias, innovador y a medida del interior del SOB (Porras, et al 2009).

La Universidad dicta en la actualidad, numerosas carreras con un perfil emprendedor (Tabla 1), con la finalidad de formar nuevos actores, que al margen de buscar empleo tengan la capacidad para generar su propio espacio laboral, contribuyendo a fomentar en los estudiantes la permanencia en sus lugares de origen (una de las principales problemáticas que plantea el SOB), y a transformarse en motores de desarrollo local (Porras, et al, 2009).

Frente a ese desafío, muchos autores coinciden en la necesidad de emplear metodologías y actividades pedagógicas que rompan con el esquema tradicional de la clase magistral, utilizando entonces diversas estrategias donde el participante tenga una postura más activa en el proceso de aprendizaje. (Oliveras, 2015; Guglielmi Ovalles, et al, 2013).

Para contribuir al logro de los objetivos planteados por la UPSO, como docentes del área de Emprendedorismo y Administración, empezamos a investigar sobre nuevas herramientas pedagógicas para introducir en el aula, considerando que, tal como lo plantea María Acaso en su libro RedEvolution: "no siempre lo que uno enseña es lo que el estudiante aprende" (2013, p.25). El objetivo buscado era, además de fortalecer los conceptos teóricos, hacer de cada clase un encuentro de discusión y aprendizaje, fortaleciendo ciertas competencias esenciales tanto para emprender como para gestionar una MiPyME. Entre varias ideas, notamos que el método del caso podía resultar una herramienta útil para recrear la realidad de una empresa en el aula, pues implica precisamente eso. Asimismo, en general en la UPSO se trabaja con grupos reducidos de alumnos, cuestión que favorece la aplicación de la metodología.

De acuerdo con la bibliografía, enseñar a partir del análisis de un caso, facilita en los estudiantes el desarrollo de competencias para examinar problemas complejos de un modo crítico, logrando aplicar sus conocimientos cuando analizan las ideas. Se promueve la comprensión, y que los estudiantes "apliquen lo que saben" cuando analizan los datos y/o proponen soluciones o respuestas. Facilita el aprendizaje basado en la experiencia y motiva a usar sentimientos, actitudes y valores aparte de la información (Guglielmi Ovalles, et al, 2013). Las respuestas no siempre son únicas, ni perfectas, lo que también da la posibilidad de escuchar y comunicar entre pares, cuestión que los prepara para enfrentar situaciones en la vida real. En suma, se puede afirmar que a través del método del caso se enriquece la formación y el proceso de aprendizaje, fomentando el trabajo en equipo, la toma de decisiones, así como el desarrollo competencias y habilidades tales como el análisis, la síntesis y la evaluación de la información.

Lo cierto es que, a la hora de buscar dichos casos, numerosos libros sobre administración y creación de empresas presentan ejemplos de firmas grandes y exitosas, ubicadas en un contexto muy diferente al del SOB y al de nuestros estudiantes, e incluso la mayoría de los ejemplos se encuentran situados territorialmente fuera del radio de influencia de la

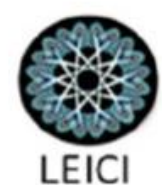


Universidad, aspecto que dificulta en ellos la identificación con la empresa analizada, sintiéndose ajenos a ella.

Tabla 1: Carreras de la Universidad Provincial del Sudoeste

\begin{tabular}{|c|c|}
\hline LICENCIATURAS EN: & $\begin{array}{ll} & \text { Gerenciamiento de PyMEs } \\
- & \text { Desarrollo Local y Regional } \\
- & \text { Diseño de Indumentaria y Calzado } \\
\text { - } & \text { Diseño Industrial } \\
- & \text { Política Local y Gestión Pública } \\
- & \text { Medicina } \\
- & \text { Enfermería }\end{array}$ \\
\hline $\begin{array}{c}\text { TECNICATURAS } \\
\text { UNIVERSITARIAS (TU) } \\
\text { EN: }\end{array}$ & $\begin{array}{ll}- & \text { Creación y Gestión de PyMes } \\
- & \text { Emprendimientos Turísticos } \\
- & \text { Emprendimientos Audiovisuales } \\
- & \text { Emprendimientos del Diseño } \\
- & \text { Emprendimientos Agropecuarios } \\
- & \text { Gestión Cultural y Emprendimientos Culturales } \\
- & \text { Emprendimientos Agroalimentarios } \\
- & \text { Asuntos Municipales } \\
- & \text { Emprendimientos Informáticos Periodismo y Emprendimientos de la } \\
& \text { Comunicación } \\
- & \text { Gestión del Comercio Exterior y Régimen Aduanero } \\
- & \text { Acompañamiento Terapéutico } \\
- & \text { Gestión Ambiental } \\
- & \text { Gestión de Emprendimientos Deportivos } \\
- & \text { Manejo y Comercialización de Granos }\end{array}$ \\
\hline $\begin{array}{c}\text { DIPLOMATURAS } \\
\text { UNIVERSITARIAS (DU) } \\
\text { EN: }\end{array}$ & $\begin{array}{ll}- & \text { Gestión del Comercio Exterior } \\
- & \text { Asuntos Municipales y Gestión Local } \\
- & \text { Desarrollo Local } \\
- & \text { Gestión Ambiental } \\
- & \text { Gestión Administrativa } \\
- & \text { Agronegocios y Desarrollo Productivo } \\
- & \text { Locución } \\
- & \text { Acompañante Terapéutico } \\
- & \text { Guía Universitario de Turismo } \\
- & \text { Técnicas Audiovisuales }\end{array}$ \\
\hline $\begin{array}{c}\text { CARRERAS } \\
\text { PROFESIONALES }\end{array}$ & $\begin{array}{l}\text { - } \\
\text { _ }\end{array}$ \\
\hline $\begin{array}{c}\text { CICLOS DE } \\
\text { COMPLEMENTACIÓN } \\
\text { CURRICULAR (CCC): }\end{array}$ & $\begin{array}{l}\text { - de la Licenciatura en Educación Física y Gestión de Emprendimientos } \\
\text { Deportivos }\end{array}$ \\
\hline
\end{tabular}

Fuente: www.upso.edu.ar

Estas cuestiones nos motivaron a pensar en la posibilidad de comenzar a redactar casos de empresas reales con problemas reales que tuvieran que ver con nuestro contexto: el SOB, 
abriendo la posibilidad de que los estudiantes pudiesen trabajar no sólo con ejemplos de su propia localidad, sino también con casos de otras ciudades, situadas a su alrededor.

A fin de compartir nuestra experiencia, en el presente trabajo presentamos las principales actividades llevadas a cabo para alcanzar el objetivo planteado, así como los resultados obtenidos. Para ello, en primera medida realizamos un breve marco referido al método del caso, sus características y potencialidades. Seguidamente comentamos la metodología aplicada, y finalmente, describimos el principal resultado obtenido: el desarrollo de un material didáctico propio.

\section{Marco teórico}

\subsection{Concepto del método del caso}

De acuerdo con Labrador y Andreu (2008) un caso es un vehículo o herramienta por medio de la cual se lleva al aula una problemática real para que estudiantes y profesores examinen a conciencia una situación planteada y desarrollen, a través de la discusión que se genera, conocimientos y habilidades, de acuerdo con los objetivos específicos de la sesión y generales de cualquier asignatura. Se pretende acercar a los estudiantes la posibilidad de actuar en sus posibles ámbitos de desempeño profesional.

Utiliza como punto de partida la descripción de una situación real que es analizada y discutida a partir de lineamientos impartidos por el docente. El análisis parte de preguntas críticas, que obligan a los estudiantes a identificar ideas importantes y situaciones problemáticas. Dicho análisis se puede desarrollar en grupos pequeños de discusión y esto enriquece aún más la enseñanza, al tener que respetar opiniones diversas, percibir puntos de vista diferentes, distintos roles, que no hubieran surgido trabajando de manera individual. (Wassermann, 1994).

Se puede decir que promueve la utilización de estrategias que permiten a cada estudiante construir un aprendizaje significativo, contraponiéndose a la tradicional clase magistral a través de la cual la comunicación es unidireccional, donde el docente es quien "tiene el conocimiento", y el estudiante quien "debe absorberlo".

Se intenta que las respuestas a las consignas utilizadas para el análisis no siempre sean únicas, ni perfectas (Guglielmi Ovalles et al, 2013), promoviendo el aprendizaje de comunicar sus ideas eficazmente y aceptar la ambigüedad, cuestión que los prepara para enfrentar situaciones en la vida real.

\subsection{Beneficios del método del caso}

La bibliografía específica establece que, a través del método del caso, los estudiantes pueden mejorar de manera significativa sus habilidades de gestión y fortalecer sus competencias ya que brinda la posibilidad de discutir situaciones reales en el aula, achicando la brecha existente entre la teoría y la realidad. En otras palabras, se pueden evaluar

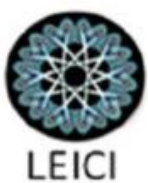


situaciones reales y aplicar a su vez diversos conceptos, en lugar de aprenderlos desde un enfoque netamente teórico.

Desde lo comportamental, también facilita en los estudiantes poner en acción diferentes competencias a través de la interacción entre los miembros del grupo. Por último, y no menos importante, algunos autores (Labrador y Andreu, 2008) se animan a afirmar que los estudiantes le terminan dedicando voluntariamente más tiempo a trabajar sobre la resolución del caso que a estudiar la teoría, ya que les resulta más interesante analizar ejemplos reales que recibir lecciones puramente teóricas.

A partir de lo anterior, se podría afirmar que se genera un aprendizaje significativo, es decir, un aprendizaje que relaciona los nuevos conceptos, con los que ya posee, y construye el conocimiento, a partir de la síntesis de ambas informaciones, a través del proceso de aprendizaje.

\subsection{Tipos de casos}

De acuerdo con Labrador y Andreu (2008) existen tres tipos:

- Los casos-problema o casos-decisión. Se trata de la descripción de una situación problemática sobre la cual es preciso tomar una decisión. En la lectura del caso se observa que la situación es interrumpida justo antes de la toma de decisión, presentando todos los datos necesarios para su análisis.

- Los casos-evaluación. Esta tipología redacta situaciones, en las que se debe evaluar el impacto de situaciones generadas por la empresa. Aquí se pueden incluir, por ejemplo, los accidentes medioambientales en los que se trata de evaluar el impacto generado y su alcance.

- Los casos-ilustración. Se trata de una situación que va más allá de la toma de decisiones, en la que se analiza un problema real y la solución que se adoptó atendiendo al contexto; lo que permite al grupo aprender sobre la forma en que una determinada organización o profesional ha tomado una decisión y el impacto de la misma.

Más allá de estas tres tipologías, consideramos que este método también puede servir para una cuarta tipología, como es el caso-aplicación. Con el caso aplicación, se intenta llevar al aula la descripción de una empresa real, con todas sus características, a fin de que los estudiantes puedan reflexionar sobre los aspectos teóricos en un caso real. No necesariamente se trata de tomar una decisión o de evaluar una decisión tomada por el empresario en un contexto dado, sino de vincular la teoría con la práctica. El caso-aplicación puede ser usado tanto para el desarrollo de la clase teórica como para cerrar el tema una vez trabajado en el aula, de modo de afianzar aún más los lineamientos impartidos.

\subsection{Formas de aplicarlo en el aula}

Para implementar el método del caso, es importante que tanto el profesor como los estudiantes estén preparados para su desarrollo. El profesor, debe preguntarse, para qué o con qué objetivo se quiere introducir el método del caso. Eso le permite decidir respecto a qué

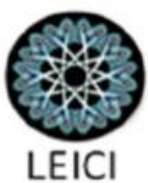


tipología aplicar. En el aula, lo recomendable es que el grupo se divida en equipos para trabajar. Se sugiere que se lea primero de manera individual y luego grupal. Siguiendo a Labrador y Andreu (2008) el trabajo en grupo fomenta el diálogo y el pensamiento crítico de sus miembros, por eso se espera que el aprendizaje de los estudiantes no sea sólo de conocimientos, sino también de habilidades y actitudes.

Cuando se opte por situaciones que los estudiantes analicen, juzguen y tomen decisiones, es bueno saber que las acciones que adopten no serán consideradas ni buenas ni malas. Tomará protagonismo la justificación realizada.

\section{Metodología de trabajo}

Convencidas de que el método del caso resulta ser una de las metodologías adecuadas para romper con el esquema tradicional de las clases magistrales, y buscando un rol más activo en el proceso de aprendizaje de los estudiantes, debíamos ahora enfrentar los siguientes desafíos:

- Conseguir casos de MiPyMEs, ya que varios de los disponibles en los libros, tienen que ver con grandes empresas exitosas;

- Lograr que esas MiPyMEs estén situadas en el interior del SOB, ya que el ecosistema empresarial es muy diferente al de las grandes metrópolis;

- Vincular las MiPyMEs elegidas con las orientaciones de las carreras impartidas por la UPSO. La Institución valora que el docente pueda relacionar su materia con el perfil de la carrera a la cual pertenece. Por ejemplo, no es la misma orientación de una asignatura como Comercialización, si se dicta para la carrera de Martillero Público que si se dicta para los Lic. en Gerenciamiento de PyMEs.

Para poder redactar cada caso, nos contactamos con diferentes organizaciones, buscamos en Internet, hicimos visitas y fuimos de a poco recopilando la información, para luego de un período de análisis y de sistematización, redactar cada ejemplo.

A fin de testear los primeros casos, los incorporamos como material de cátedra en forma de trabajos prácticos, y como forma de evaluación en los exámenes parciales y finales. De ese modo, poco a poco, notamos cómo los estudiantes se involucraban más con las asignaturas e incorporaban de una manera más amena los contenidos que impartíamos.

Una vez redactados varios casos, pensamos en la posibilidad de compilarlos en formato de libro. Este esquema nos iba a permitir que otros docentes pudieran usarlos como herramienta o como fuente de inspiración para crear nuevos ejemplos que se adecúen a la realidad de los estudiantes y de su contexto educativo.

Con el objetivo de enriquecer el compendio de casos, convocamos a otros docentes del área a redactar los propios. Al compartir la idea, algunos se sumaron a la propuesta y comenzaron la búsqueda de MiPyMEs para elaborar sus ejemplos y completar con nuevos casos el material. De esta manera recopilamos 13 casos de MiPyMEs del SOB, con formato de libro digital. 


\section{Resultados}

Luego de meses de trabajo, obtuvimos el libro de casos titulado "Acercando la empresa a la Universidad: Casos de MiPyMEs como herramienta pedagógica para utilizar en el aula". El libro se divide en tres apartados. En el primero se realiza una breve descripción de la herramienta, explicando de qué se trata, qué tipos de casos existen, y cómo aplicarlos en el aula. En el segundo se presentan los casos seleccionados. Aportamos ejemplos de diversas localidades del sudoeste bonaerense, desde micro emprendimientos hasta Pymes ya consolidadas, tanto del sector productivo como del de servicios, unipersonales como asociativos, incluyendo también empresas familiares. El abanico logra cubrir varias de las orientaciones emprendedoras de las carreras de la UPSO. Esto se sintetiza en la Tabla 2.

Tabla 2: Características de las empresas elegidas

\begin{tabular}{|c|c|c|c|c|}
\hline $\begin{array}{l}\text { Nombre de la } \\
\text { Empresa }\end{array}$ & Localidad & Breve descripción & Tipología & $\begin{array}{c}\text { Carreras con las que se } \\
\text { vincula }\end{array}$ \\
\hline Casa Maci & $\begin{array}{l}\text { General La } \\
\text { Madrid }\end{array}$ & $\begin{array}{l}\text { Es una casa de comidas } \\
\text { tradicional de la } \\
\text { localidad, que adicionó } \\
\text { un supermercado. }\end{array}$ & Empresa familiar. & $\begin{array}{l}\text { - } \quad \text { Lic. en Gerenciamiento } \\
\text { de PyMEs. } \\
\text { - } \quad \text { TU Creación y Gestión de } \\
\text { PyMes } \\
\text { - } \quad \text { DU en Gestión } \\
\text { Administrativa }\end{array}$ \\
\hline Le Utopik & $\begin{array}{l}\text { Coronel } \\
\text { Suárez }\end{array}$ & $\begin{array}{c}\text { Se trata de un taller que } \\
\text { fabrica zapatillas y vende } \\
\text { a todo el país. }\end{array}$ & $\begin{array}{l}\text { Emprendimiento } \\
\text { unipersonal }\end{array}$ & $\begin{array}{ll}\text { - } & \text { Lic. en Gerenciamiento } \\
\text { de PyMEs. } \\
\text { - } \\
\text { TU Creación y Gestión de } \\
\text { PyMes } \\
\text { - } \quad \text { DU en Gestión } \\
\text { Administrativa } \\
-\quad \text { Lic. en Diseño industrial. } \\
-\quad \text { Lic. en Diseño de } \\
\text { indumentaria } \\
\text { - TU en emprendimientos } \\
\text { del diseño. }\end{array}$ \\
\hline $\begin{array}{l}\text { Curves Bahía } \\
\text { Blanca }\end{array}$ & $\begin{array}{l}\text { Bahía } \\
\text { Blanca }\end{array}$ & $\begin{array}{l}\text { Es un gimnasio puesto en } \\
\text { marcha a través de una } \\
\text { franquicia proveniente de } \\
\text { Estados Unidos. }\end{array}$ & Empresa familiar. & 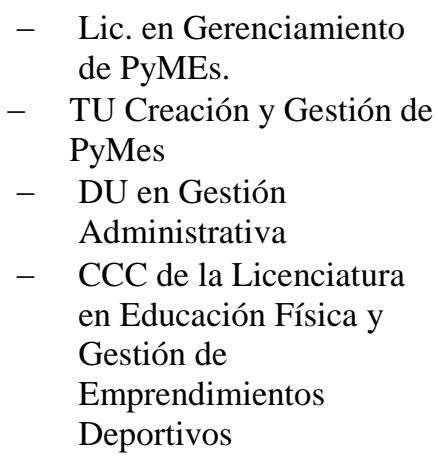 \\
\hline Stalla \& Co & $\begin{array}{l}\text { Coronel } \\
\text { Suárez }\end{array}$ & $\begin{array}{l}\text { Es un taller de calzado } \\
\text { que fabrica para varias }\end{array}$ & Empresa familiar. & $\begin{array}{l}\text { - Lic. en Gerenciamiento } \\
\text { de PyMEs. }\end{array}$ \\
\hline
\end{tabular}




\begin{tabular}{|c|c|c|c|c|}
\hline & & $\begin{array}{l}\text { marcas del país, y a su } \\
\text { vez desarrolló su marca } \\
\text { propia. }\end{array}$ & & $\begin{array}{ll}\text { - } & \text { TU Creación y Gestión de } \\
& \text { PyMes } \\
- & \text { DU en Gestión } \\
& \text { Administrativa } \\
- & \text { Lic. en Diseño industrial. } \\
- & \text { Lic. en Diseño de } \\
& \text { indumentaria } \\
-\quad & \text { TU en emprendimientos } \\
& \text { del diseño.. }\end{array}$ \\
\hline $\begin{array}{l}\text { Establecimien } \\
\text { to El Tata }\end{array}$ & Puan & $\begin{array}{l}\text { Se trata de un tambo de } \\
\text { pocos años de } \\
\text { antigüedad. }\end{array}$ & Empresa familiar. & 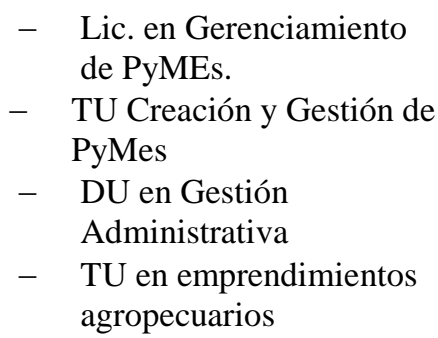 \\
\hline Ferreira Sport & $\begin{array}{l}\text { Bahía } \\
\text { Blanca }\end{array}$ & $\begin{array}{c}\text { Es una empresa que } \\
\text { vende indumentaria } \\
\text { deportiva y accesorios, y } \\
\text { se ha expandido con el } \\
\text { correr de los años a } \\
\text { varias localidades de la } \\
\text { región. }\end{array}$ & Empresa familiar. & 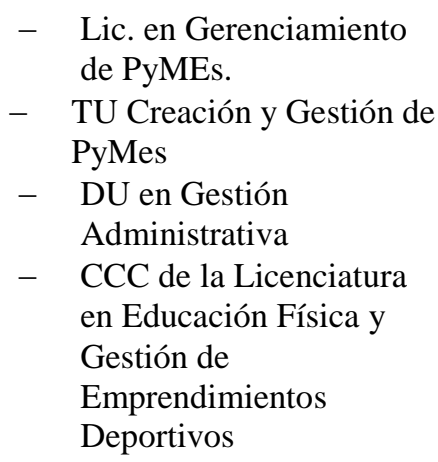 \\
\hline Serpor SA & Ing. White & $\begin{array}{l}\text { Se trata de una Pyme que } \\
\text { presta servicios de } \\
\text { mantenimiento, logística } \\
\text { e ingeniería civil. }\end{array}$ & Empresa familiar. & $\begin{array}{l}\text { - } \quad \text { Lic. en Gerenciamiento } \\
\text { de PyMEs. } \\
\text { - } \quad \text { TU Creación y Gestión de } \\
\text { PyMes } \\
\text { - DU en Gestión } \\
\text { Administrativa }\end{array}$ \\
\hline $\begin{array}{l}\text { Swing } \\
\text { Helados }\end{array}$ & $\begin{array}{c}\text { Cnel. } \\
\text { Dorrego }\end{array}$ & $\begin{array}{c}\text { Es una fábrica de helados } \\
\text { que se está expandiendo } \\
\text { por la región. }\end{array}$ & Empresa familiar & $\begin{array}{l}\text { - } \quad \text { Lic. en Gerenciamiento } \\
\text { de PyMEs. } \\
\text { - } \quad \text { TU Creación y Gestión de } \\
\text { PyMes } \\
\text { - DU en Gestión } \\
\text { Administrativa }\end{array}$ \\
\hline $\begin{array}{l}\text { H2O Aqua } \\
\text { Gym }\end{array}$ & $\begin{array}{l}\text { Bahía } \\
\text { Blanca }\end{array}$ & $\begin{array}{l}\text { Es el caso de una } \\
\text { empresa que inicialmente } \\
\text { fue un natatorio de Bahía } \\
\text { Blanca y hoy en día se ha } \\
\text { convertido en un }\end{array}$ & Empresa familiar & $\begin{array}{l}\text { - } \quad \text { Lic. en Gerenciamiento } \\
\text { de PyMEs. } \\
\text { - } \quad \text { TU Creación y Gestión de } \\
\text { PyMes } \\
\text { - DU en Gestión }\end{array}$ \\
\hline
\end{tabular}




\begin{tabular}{|c|c|c|c|c|}
\hline & & $\begin{array}{l}\text { referente del Fitness } \\
\text { bahiense. }\end{array}$ & & $\begin{array}{l}\text { Administrativa } \\
\text { - CCC de la Licenciatura } \\
\text { en Educación Física y } \\
\text { Gestión de } \\
\text { Emprendimientos } \\
\text { Deportivos }\end{array}$ \\
\hline Mate Sabio & $\begin{array}{l}\text { Bahía } \\
\text { Blanca }\end{array}$ & $\begin{array}{l}\text { Se trata de un invento } \\
\text { bahiense hoy } \\
\text { ampliamente difundido } \\
\text { en toda la Argentina. }\end{array}$ & Empresa familiar. & $\begin{array}{ll}\text { - } & \text { Lic. en Gerenciamiento } \\
\text { de PyMEs. } \\
\text { - } \\
\text { TU Creación y Gestión de } \\
\text { PyMes } \\
\text { - } \quad \text { DU en Gestión } \\
\text { Administrativa } \\
-\quad \text { Lic. en Diseño industrial. } \\
-\quad \text { TU en emprendimientos } \\
\text { del diseño. }\end{array}$ \\
\hline Mamma Mia! & Médanos & $\begin{array}{c}\text { Es el caso de un } \\
\text { emprendimiento de } \\
\text { carteras de una alumna } \\
\text { de la UPSO de la } \\
\text { localidad de Médanos. }\end{array}$ & $\begin{array}{l}\text { Emprendimiento } \\
\text { unipersonal }\end{array}$ & $\begin{array}{ll}- & \text { Lic. en Gerenciamiento } \\
& \text { de PyMEs. } \\
- & \text { TU Creación y Gestión de } \\
& \text { PyMes } \\
- & \text { DU en Gestión } \\
& \text { Administrativa } \\
- & \text { Lic. en Diseño industrial. } \\
- & \text { Lic. en Diseño de } \\
& \text { indumentaria } \\
-\quad & \text { TU en emprendimientos } \\
& \text { del diseño. }\end{array}$ \\
\hline $\begin{array}{l}\text { Viñas de } \\
\text { Lucía }\end{array}$ & $\begin{array}{c}\text { C. } \\
\text { Patagones }\end{array}$ & $\begin{array}{l}\text { Se trata de un pequeño } \\
\text { viñedo con potencial en } \\
\text { la región. }\end{array}$ & Empresa familiar & $\begin{array}{ll}\text { - } & \text { Lic. en Gerenciamiento } \\
\text { de PyMEs. } \\
-\quad \text { TU Creación y Gestión de } \\
\text { PyMes } \\
-\quad \text { DU en Gestión } \\
\text { Administrativa } \\
-\quad \text { TU en emprendimientos } \\
\text { agropecuarios } \\
-\quad \begin{array}{l}\text { TU en emprendimientos } \\
\text { agroalimentarios }\end{array}\end{array}$ \\
\hline Muñecas & $\begin{array}{l}\text { Bahía } \\
\text { Blanca }\end{array}$ & $\begin{array}{l}\text { Es el caso de un } \\
\text { emprendimiento de } \\
\text { calzado de diseño. }\end{array}$ & Empresa familiar & $\begin{array}{ll}- & \text { Lic. en Gerenciamiento } \\
\text { de PyMEs. } \\
-\quad \text { TU Creación y Gestión de } \\
\text { PyMes } \\
-\quad \text { DU en Gestión } \\
& \text { Administrativa } \\
-\quad & \text { Lic. en Diseño industrial. } \\
- & \text { Lic. en Diseño de } \\
& \text { indumentaria } \\
-\quad \text { TU en emprendimientos }\end{array}$ \\
\hline
\end{tabular}




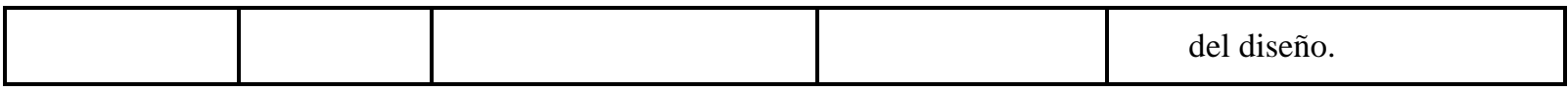

Fuente: elaboración propia

Por último, el libro incluye modelos de trabajos prácticos para asignaturas del área de Administración y Gestión de MiPyMEs, diseñados en función de la lectura de alguno de los casos. Se incluye además una sugerencia de resolución y la bibliografía de referencia a fin de que los docentes puedan acudir a ella en caso de resultarles necesario.

La labor realizada durante todos estos meses de trabajo, fue desarrollada para que pueda ser efectivamente utilizada en el aula. De las experiencias recopiladas, a partir de su aplicación, esperamos en un futuro enriquecer el material didáctico, así como completar el espectro de áreas de los casos, incluyendo sectores como el de organizaciones turísticas, audiovisuales e inmobiliarias para cubrir áreas de carreras aun no incluidas.

\section{Conclusiones}

La metodología del estudio de caso constituye una herramienta pedagógica valiosa para la enseñanza del emprendedorismo y la gestión de MiPyMEs. La misma permite no sólo la aplicación e incorporación de conceptos teóricos vinculados a la creación y gestión, sino también habilidades y actitudes de utilidad para cualquier estudiante, tanto para llevar a cabo su emprendimiento personal como para su desarrollo personal como profesional.

A partir de esta experiencia presentada, podemos reflexionar que como docentes, es posible innovar y transformar las herramientas que tenemos a nuestro alcance para cumplir nuestra labor, adecuando el dictado de las asignaturas, al contexto y a la realidad a al cual se va a aplicar. Una de las formas de lograrlo, es a partir de la elaboración de un material didáctico que contribuya al aprendizaje de los estudiantes, permitiéndoles identificarse a partir del modelo de rol, con la realidad en la que se desempeñan.

A partir de los casos de MiPyMEs compilados en la edición de "Acercando la empresa a la Universidad: Casos de MiPyMEs como herramienta pedagógica para utilizar en el aula", podemos afirmar que los docentes de la UPSO disponen de distintas propuestas de casos para trabajar en las diversas localidades y a su vez, aptos para ser adaptados a la orientación de la carrera a la cual pertenece la asignatura.

Como futuras líneas de trabajo, nos resta procesar las experiencias a partir de la aplicación del material desarrollado, incorporar casos pertenecientes a áreas aun no cubiertas, y también analizar la percepción por parte de los alumnos, respecto a la utilidad de la aplicación del método del caso como herramienta. Los resultados obtenidos contribuirán para mejorar cada día nuestra tarea.

\section{Referencias}

Acaso, M. (2013). Reduvolution. Madrid: Pai-dos IBERICA.

Guglielmi, I., Valduz, Q., \& Beatriz, Y. (2013). El estudio de caso como herramienta pedagógica en la formación emprendedora. Acción Pedagógica, 22(1), 132-141.

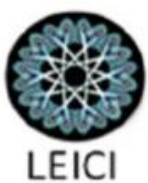


Labrador-Piquer, M. J., \& Andreu-Andrés, M. A. (2008). Metodologías activas. Valencia: Editorial UPV. Obtenido el 20 de octubre de 2016.

Porras, E.; Oliveras, G.; Vigier, H.; Savoretti, A., Dichiara, R.; Bruno, M. (2009, diciembre). La formación de emprendedores y el fomento a la iniciativa empresarial: la experiencia de la Universidad Provincial del Sudoeste. Ponencia presentada en las $\mathrm{I}^{\circ}$ Jornada sobre PYME e Iniciativa Empresarial. Universidad Carlos III, Getafe, Madrid, España.

Wassermann, S. (1994). El estudio de casos como método de enseñanza. Amorrortu. 